\title{
Fragmented QRS Predicts the Arrhythmic Events in Patients with Heart Failure Undergoing ICD Implantation for Primary Prophylaxis: More Fragments More Appropriate ICD Shocks
}

Firat Özcan, M.D., Osman Turak, Uğur Canpolat, M.D., Sedat Avci, M.D., Derya Tok, M.D., Ahmet Isleyen, M.D., Muhammed Cebeci, M.D., Hüseyin Yuzgeçer, M.D., Özgül Malçok Gurel, M.D., Serkan Topaloglu, M.D., Dursun Aras, M.D., Fatma Nurcan Basar, M.D., and Sinan Aydogdu, M.D.

From the Cardiology Clinic, Türkiye Yüksek Ihtisas Training and Research Hospital, Ankara, Turkey

Background: Fragmented QRS complex (fQRS) is associated with cardiovascular outcomes in various patient populations. Although there were clinical studies investigating the association of fQRS with arrhythmic events in patients with systolic heart failure, the results were conflicting regarding the association of implantable cardioverter defibrillator (ICD) shocks and fQRS. In this study, we aimed to evaluate the association between the presence and extent of fQRS with appropriate ICD shocks and/or all-cause mortality.

Methods: A total of 215 patients (age: $58.2 \pm 11.6$ years, $72.5 \%$ male) with the diagnosis of left ventricular systolic heart failure in whom ICD had been implanted for primary prophylaxis were enrolled. Standard ECG evaluation revealed fQRS complex in 123 patients (57.2 \%). The phenomenon of fQRS was defined as deflections at the beginning of the QRS complex, on top of the $\mathrm{R}$ wave, or in the nadir of the $\mathrm{S}$ wave similar to the definition in CAD.

Results: At mean $23.5 \pm 12.1$ months follow-up, all-cause mortality was observed in 45 $(20.9 \%)$ patients and 111 (51.6\%) patients experienced appropriate ICD shocks. Median number of ECG leads with fQRS were higher in patients with appropriate ICD shocks (3 [2-6] vs 1 [0-2], P $<0.001$, respectively). The presence of fQRS (HR: 6.64, $95 \% \mathrm{Cl}: 3.54-12.4, \mathrm{P}<0.001$ ) and the number of leads with fQRS (HR: $1.35,95 \% \mathrm{Cl}: 1.22-1.67)$ were found as independent predictors of appropriate ICD shocks. Additionally, there was a negative correlation between left ventricular ejection fraction and the number of leads with fQRS $(r=-0.434, P<0.001)$. Rates of all-cause mortality did not differ between the fQRS $(+)(29[24 \%])$ and fQRS $(-)(16[17 \%])$ groups $(\mathrm{P}=$ 0.27).

Conclusion: Our findings suggest that the presence and extent of fQRS complex on standard 12-lead ECG predicts appropriate ICD shocks in patients with left ventricular systolic heart failure who underwent ICD implantation for primary prophylaxis.

Ann Noninvasive Electrocardiol 2014;19(4):351-357

$\underline{\text { Implantable cardioverter defibrillator; appropriate shock; fragmented QRS complex }}$

Implantable cardioverter defibrillator (ICD) implantation is recommended for primary prevention of sudden cardiac death to reduce total mortality in selected patients with ischemic or nonischemic cardiomyopathy. ${ }^{1}$ Although there were several studies investigating the predictors of appropriate ICD shocks in those patients, left ventricular ejection fraction (LVEF) seems to be significant major

Address for correspondence: Uğur Canpolat, M.D., Türkiye Yüksek Ihtisas Training and Research Hospital, Cardiology Clinic, Sihhiye, 06100, Ankara,Turkey.Fax: +90 312306 1134; E-mail:dru_canpolat@yahoo.com

This study was not funded by any institution. Authors do not have any conflict of interest. 
contributor in heart failure with either ischemic ${ }^{2}$ or nonischemic etiology. ${ }^{3}$ Fragmented QRS complex (fQRS), as a noninvasive prognostic marker on resting electrocardiography (ECG) emerged as a diagnostic and/or prognostic marker in several cardiovascular conditions. ${ }^{4-7}$ However, clinical trials showed conflicting results regarding the association of arrhythmic events and appropriate ICD shocks with fQRS in patients with systolic heart failure. ${ }^{8-13}$ So, in this study, we aimed to investigate the association of appropriate ICD shocks and all-cause mortality with the presence and extent of fQRS in patients with systolic heart failure who underwent ICD implantation for primary prophylaxis previously.

\section{METHODS}

\section{Study Population}

A total of 215 eligible patients (mean age: $58.2 \pm$ 11.6 years, $72.5 \%$ male) who admitted to our Pacemaker Clinic for scheduled ICD interrogation as a part of routine follow-up visits between June 2011 and April 2012 were enrolled in this study. All patients had primary prevention indications according to the current American College of Cardiology/American Heart Association Guidelines for ICD at the time of implantation. The study population included patients with $>18$ years old, with dilated cardiomyopathy due to ischemic or nonischemic etiology, chronic stable heart failure (NYHA class I-III) and with LVEF $<35 \%$. Patients in whom ICD implantation have been performed for secondary prophylaxis were excluded from the study. The study population divided into two subgroups according to the detection of appropriate ICD shocks (Group I; with appropriate ICD shocks and Group II; without any ICD shocks). Informed consent was taken from each patient before enrollment. The study was in compliance with the principles outlined in the Declaration of Helsinki and approved by Institutional Ethics Committee.

\section{Electrocardiography}

At the time of initial diagnosis, standard resting 12-lead ECGs (filter range $0.15-100 \mathrm{~Hz}$, AC filter $60 \mathrm{~Hz}, 25 \mathrm{~mm} / \mathrm{s}, 10 \mathrm{~mm} / \mathrm{mV}$ ) were analyzed by two independent readers blinded to the patient clinical and echocardiographic findings. There was 96.5\% concordance for the ECG signs including
fQRS. When there was a disagreement, the final conclusion was achieved by mutual agreement.

Fragmentation of narrow QRS $(<120 \mathrm{~ms})$ is defined as presence of an additional $R$ wave $\left(R^{\prime}\right)$ or notching in the nadir of the $\mathrm{S}$ wave, or the presence of $>1 \mathrm{R}^{\prime}$ in two contiguous leads, corresponding to a major coronary artery territory on the resting 12 lead ECG (Fig. 1). Fragmentation of wide complex QRS (>120 ms) consists of various RSR patterns, with more than $2 \mathrm{R}$ waves $\left(\mathrm{R}^{\prime \prime}\right)$ or more than two notches in the $\mathrm{R}$ wave, or more than two notches in the downstroke or upstroke of the S wave. ${ }^{14}$

\section{Echocardiography}

Standard echocardiographic imaging was performed in the left lateral decubitus position in the parasternal and apical views. Twodimensional, M-mode, pulsed and color flow Doppler echocardiographic examinations of all subjects were performed by the same examiner with a commercially available machine (Vivid 7, GE Medical System, Horten, Norway; $3.5-\mathrm{MHz}$ phased array transducer). LVEF was calculated from apical 4-chamber views, according to the modified Simpson's rule. ${ }^{15}$

\section{Follow-up and ICD Interrogation}

The end points for the study were all-cause mortality, appropriate ICD shocks due to ventricular tachyarrhythmias and combined endpoint of appropriate ICD shocks or all-cause mortality. Outpatient follow-up visits were performed for every 6 months or according to the patient's clinical status. All stored episodes of arrhythmia that occurred since the implantation of the device were retrospectively analyzed for accuracy of detection, diagnostic precision, and appropriateness of device therapy. Device interrogation was performed by an experienced electrophysiologist who was unaware of the presence of fQRS. The ICD programming included therapy for standard ventricular tachycardia (VT) with antitachycardia pacing combined with low-energy shock therapy and for ventricular fibrillation (VF) shock therapy with a 300-ms cutoff cycle interval. It was considered standard VT in the presence of sustained tachycardia with a cycle interval ranging from 300 to $400 \mathrm{~ms}$, not discriminated as supra-VT by specific algorithms. It was considered VF when the cycle interval was inferior to $300 \mathrm{~ms}$. Appropriate ICD shock was 

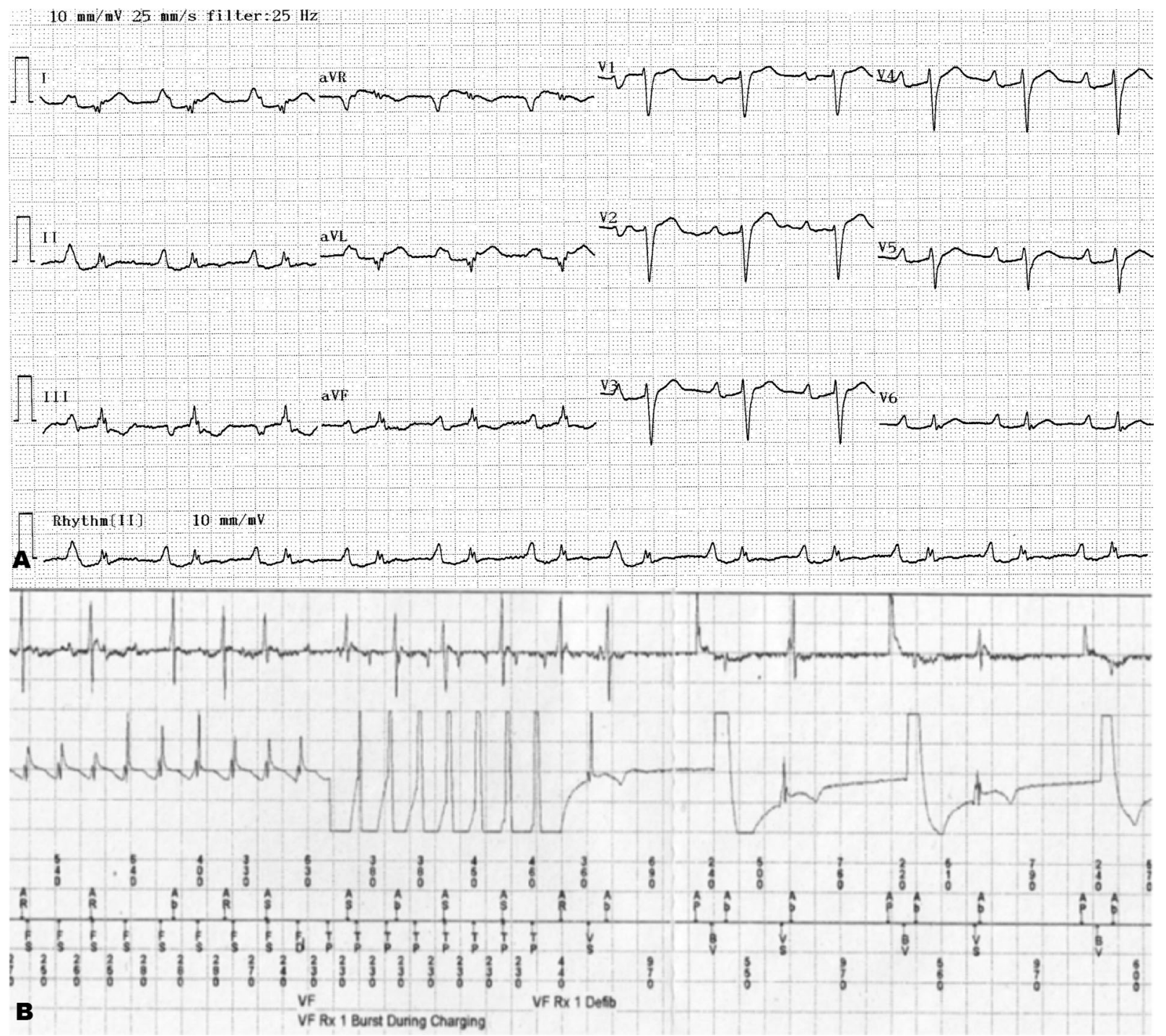

Figure 1. (A) The electrocardiogram of a patient with left ventricular ejection fraction of $28 \%$ and fQRS in inferolateral derivations (six leads with fQRS) in whom most frequent ventricular tachycardia episodes were determined during ICD interrogation (B).

defined as therapy for rapid sustained ventricular tachycardia or fibrillation episode.

\section{Statistical Analysis}

Continuous variables were expressed as mean \pm $\mathrm{SD}$ or median (interquartle range) and categorical variables were expressed as percentages. Continuous variables were tested for normal distribution by the Kolmogorov-Smirnov test. Categorical variables were analyzed by chi-square test or Fisher's exact test. Comparisons of continuous variables between the two groups were performed using the Student's t-test. Kappa statistics was used to evaluate the interobserver agreement of ECG signs. Univariate and multivariable Cox proportional hazard regression was applied for assessment of the association of baseline clinical and noninvasive variables with occurrence of arrhythmic events. Kaplan-Meier estimator was generated to estimate survival curves and log-rank test was used to compare survival probability among the f-QRS $(+)$ and fQRS (-) groups. Statistical analyses were performed using SPSS statistical software (version 20.0; SPSS Inc., Chicago, IL, USA). A P value $<0.05$ was considered statistically significant. 
354 • A.N.E. • July 2014 • Vol. 19, No. 4 • Özcan, et al. • fQRS and Appropriate ICD Shocks

Table 1. Baseline Characteristics of the Study Population due to Development of Appropriate ICD Shocks ( $\mathrm{n}=$ 215)

\begin{tabular}{|c|c|c|c|}
\hline & Shock $(-) n=104$ & Shock $(+) n=111$ & $\mathbf{P}$ \\
\hline \multicolumn{4}{|l|}{ Clinical parameters } \\
\hline Age, years & $56.6 \pm 12.8$ & $59.8 \pm 12.6$ & 0.001 \\
\hline Male gender, n (\%) & 79 (76 \%) & 77 (64\%) & 0.279 \\
\hline Hypertension, n (\%) & $70(67 \%)$ & $84(76)$ & 0.174 \\
\hline Dyslipidemia, n (\%) & $62(60 \%)$ & $73(66 \%)$ & 0.351 \\
\hline DM, n (\%) & $21(21 \%)$ & $25(23 \%)$ & 0.677 \\
\hline Smoking, n (\%) & $15(14 \%)$ & $17(15 \%)$ & 0.854 \\
\hline Ischemic etiology, n (\%) & $42(41 \%)$ & $60(59 \%)$ & 0.045 \\
\hline \multicolumn{4}{|l|}{ NYHA functional class } \\
\hline I & $39(51 \%)$ & 37 (49\%) & \\
\hline II & $53(47 \%)$ & $59(53 \%)$ & 0.787 \\
\hline III & $12(44 \%)$ & $15(56 \%)$ & \\
\hline \multicolumn{4}{|l|}{ Laboratory findings } \\
\hline FPG, $\mathrm{mg} / \mathrm{dL}$ & 102(94-117) & $104(93-134)$ & 0.669 \\
\hline Serum creatinine, mg/dL & $0.95(0.8-1.2)$ & $1.0(0.9-1.2)$ & 0.441 \\
\hline Free T3, pg/mL & $2.7 \pm 0.7$ & $2.9 \pm 0.8$ & 0.461 \\
\hline Free $\mathrm{T} 4, \mathrm{pg} / \mathrm{mL}$ & $1.4 \pm 0.6$ & $1.3 \pm 0.3$ & 0.873 \\
\hline WBC, $\times 10^{3} / \mathrm{uL}$ & $8.2 \pm 1.7$ & $8.3 \pm 1.6$ & 0.823 \\
\hline Hemoglobin, mg/dL & $14.3 \pm 1.6$ & $13.8 \pm 1.5$ & 0.086 \\
\hline Platelet, range, $\times 10^{3} / \mathrm{uL}$ & $218(118-274)$ & 216 (188-295) & 0.232 \\
\hline $\mathrm{CRP}, \mathrm{mg} / \mathrm{dL}$ & $9.6 \pm 3.6$ & $11.1 \pm 4.0$ & 0.645 \\
\hline \multicolumn{4}{|l|}{ Electrocardiographic findings } \\
\hline Sinus rhythm, n (\%) & $74(71 \%)$ & $82(74 \%)$ & 0.655 \\
\hline Atrial fibrillation, $\mathrm{n}(\%)$ & $30(29 \%)$ & $29(26 \%)$ & \\
\hline LVEF,\%, mean & $28.1 \pm 3.7$ & $27.3 \pm 3.2$ & 0.324 \\
\hline QRS duration, msec & $100(90-120)$ & $120(100-130)$ & $<0.001$ \\
\hline No. leads with fORS & $1(0-2)$ & $3(2-6)$ & $<0.001$ \\
\hline No. patients with fQRS, $n(\%)$ & $35(34 \%)$ & $88(75 \%)$ & $<0.001$ \\
\hline
\end{tabular}

$\mathrm{CRP}=\mathrm{C}$-reactive protein; $\mathrm{DM}=$ diabetes mellitus; FPG = fasting plasma glucose; fQRS = fragmentation of ORS complex; ICD $=$ implantable cardioverter defibrillator; LVEF = left ventricular ejection fraction; NYHA $=$ New York Heart Association; WBC $=$ white blood cell.

\section{RESULTS}

During a mean follow-up of $23.5 \pm 12.1$ months, appropriate ICD shock was observed in 111 of 215 patients (51.6\%). Study population divided into two subgroups due to presence of appropriate ICD shocks (group I: 111 patients and group II: 104 patients). The baseline characteristics of the study population according to the presence of ICD shocks were shown in Table 1. All clinical and laboratory parameters were similar among study groups except older age in group I $(\mathrm{P}<0.05)$. While ECG findings were similar regarding the baseline rhythm, there were significant differences between groups due to QRS duration (100 [90-120] $\mathrm{ms}$ in group I vs 120 [100-130] ms in group II, $\mathrm{P}<0.001$ ), presence and number of leads with fQRS complex $(\mathrm{P}<0.05)$.

The predictors of appropriate ICD shocks were determined by univariable and multivariable regression analysis. The presence of fQRS (HR: 6.64, 95\% CI: 3.54-12.4, P $<0.001$ ), number of leads with fQRS (HR: 1.35, 95\% CI: 1.22-1.67) and ischemic etiology (OR: 3.04, 95\% CI: 2.93-3.15, $\mathrm{P}<0.001$ ) were found as independent predictors of appropriate ICD shocks after adjustment for other variables including age, gender, LVEF, and QRS duration (Table 2). Furthermore, Spearman correlation analysis revealed that there were significant negative correlation between LVEF and the number of leads with fQRS $(\mathrm{r}=-0.434, \mathrm{P}<0.001)$.

A total of $45(20.9 \%)$ patients died during study follow-up, of which 29 (24\%) were in the fQRS(+) group and $16(17 \%)$ were in the fQRS $(-)$ group $(\mathrm{P}=0.27)$. Also, a total of $111(51.6 \%)$ patients experienced appropriate ICD shocks due to VT/VF, of which $88(72 \%)$ were in the fQRS $(+)$ group and $23(25 \%)$ were in the fQRS $(-)$ group $(\mathrm{P}<$ 0.001) (Table 3). Figure 2 represents the rates of event-free survival on Kaplan-Meier plots between the fQRS $(+)$ and fQRS $(-)$ groups. Additionally, all-cause mortality, appropriate ICD shocks due to VT/VF and combined end points were significantly higher in patients with ischemic etiology 
Table 2. Univariate and Multivariate Regression Analysis for Evaluating the Predictors of Appropriate ICD Shocks

\begin{tabular}{|c|c|c|c|c|}
\hline \multirow[b]{2}{*}{ Parameters } & \multicolumn{2}{|c|}{ Univariate } & \multicolumn{2}{|c|}{ Multivariate } \\
\hline & HR (CI 95 \%) & $\mathbf{P}$ & HR (CI 95 \%) & $\mathbf{P}$ \\
\hline Age, years & $0.99(0.97-1.21)$ & 0.156 & - & - \\
\hline Male gender & $1.04(0.72-1.27)$ & 0.244 & - & - \\
\hline No. leads with fQRS & $1.58(1.36-1.83)$ & $<0.001$ & $1.35(-1.22-1.67)$ & 0.001 \\
\hline Presence of fQRS & 7.54 (4.08-13.9) & $<0.001$ & $6.64(3.54-12.4)$ & $<0.001$ \\
\hline QRS duration, msec & $1.02(1.0-1.03)$ & 0.001 & $1.06(0.99-1.02)$ & 0.106 \\
\hline LVEF, \% & $0.98(0.95-1.02)$ & 0.258 & - & - \\
\hline Ischemic etiology & $3.21(2.85-3.58)$ & $<0.001$ & $3.04(2.93-3.15)$ & $<0.001$ \\
\hline
\end{tabular}

$\mathrm{Cl}=$ confidence interval; fQRS $=$ fragmentation of QRS complex; ICD = implantable cardioverter defibrillator; HR = hazard ratio; $\mathrm{msec}=$ milliseconds; No. $=$ number of.

Table 3. Summary of Study Outcomes According to the Presence of fQRS Complex

\begin{tabular}{lccc}
\hline & $\begin{array}{c}\text { fQRS (-) } \\
(\mathbf{n}=\mathbf{9 2})\end{array}$ & $\begin{array}{c}\text { fQRS (+) } \\
(\mathbf{n = 1 2 3 )}\end{array}$ & $\mathbf{P}$ \\
\hline $\begin{array}{c}\text { All-cause } \\
\text { mortality, }\end{array}$ & $16(17 \%)$ & $29(24 \%)$ & 0.270 \\
$\mathrm{n}(\%)$ & & & \\
$\begin{array}{l}\text { VT/VF, n (\%) } \\
\text { Combined } \\
\text { n (\%) }\end{array}$ & $23(25 \%)$ & $88(72 \%)$ & $<0.001$ \\
\hline
\end{tabular}

fQRS = fragmented QRS complex; VF = ventricular fibrillation; VT = ventricular tachycardia

${ }^{a}$ Combined end points included VT/VF or all-cause mortality.

independent from the presence of fQRS complex (Table 4).

\section{DISCUSSION}

Our study results showed that; (i) presence of fQRS and number of leads with fQRS were well predicted the appropriate ICD shocks in patients
Table 4. Summary of Study Outcomes According to the Etiology of the Systolic Heart Failure

\begin{tabular}{|c|c|c|c|}
\hline & $\begin{array}{c}\text { Nonisch- } \\
\text { emic }(n=113)\end{array}$ & $\begin{array}{l}\text { Ischemic } \\
(n=102)\end{array}$ & $\mathbf{P}$ \\
\hline $\begin{array}{l}\text { All-cause } \\
\text { mortality, } \\
n(\%)\end{array}$ & $14(12 \%)$ & 31 (30\%) & 0.001 \\
\hline VT/VF, n (\%) & $51(45 \%)$ & $60(59 \%)$ & 0.045 \\
\hline $\begin{array}{l}\text { Combined }{ }^{a}, \\
n(\%)\end{array}$ & 55 (49\%) & 83 (81\%) & $<0.001$ \\
\hline
\end{tabular}

with systolic heart failure and, (ii) the LVEF was negatively correlated with the number of leads with fQRS.

The mechanism of fQRS on standard 12-lead ECG is speculative. It has been suggested that fQRS has occurred as a result of inhomogeneous electrical activation of the ventricles due to myocardial scar and/or ischemia and also it was
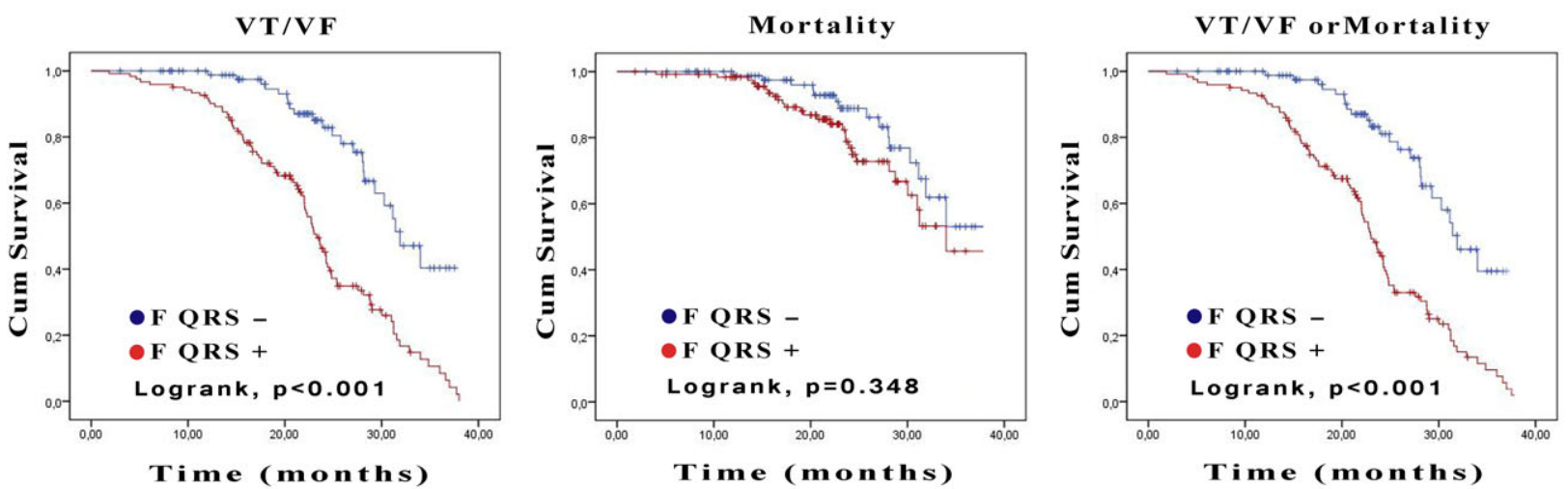

Figure 2. Kaplan-Meier analysis for the ventricular tachyarrhythmias, all-cause mortality and combined end point of all-cause mortality or ventricular tachyarrhythmia in patients with fragmented ORS and nonfragmented ORS. 
precisely predicted the arrhythmic events and sudden cardiac death in several studies. ${ }^{14,16-18}$ The diagnostic accuracy of fQRS has been investigated in different studies in which they have showed that fQRS had higher sensitivity and negative predictive value than $Q$ wave ${ }^{14}$ and fQRS was moderately sensitive but highly specific for myocardial scar. ${ }^{4}$ In imaging studies, fQRS was well signified the myocardial scar by delayed enhancement of gadolinium technique on cardiac MRI. ${ }^{19,20}$ Also several studies have confirmed the association of fQRS with myocardial perfusion abnormality and scar tissue. ${ }^{4,14,21-23}$ Endocardial and epicardial mapping in dilated cardiomyopathy patients with ventricular arrhythmias have shown that fractionated electrograms over a wide area surrounding the myocardial scar. ${ }^{24,25}$ So, we have postulated that $\mathrm{fQRS}$ precisely represents the myocardial scar which was a milieu for reentry and malignant ventricular arrhythmias.

In a study by Das et al., ${ }^{8}$ the association of arrhythmic events and mortality with fQSR was investigated in 361 patients with ischemic or nonischemic cardiomyopathy who received ICD for primary or secondary prophylaxis. At 16.6 \pm 10.2 months follow-up, fQRS was found to be a predictor of arrhythmic events, but not mortality. Moreover, electrical storm or performing VT ablation was significantly higher in the group with fQRS. Cheema et al. ${ }^{9}$ evaluated the association of fQRS with all-cause and arrhythmic mortality in 842 patients with moderate to severe left ventricular systolic dysfunction (ischemic or nonishemic etiology). During $40 \pm 17$ months follow-up period, they have found no association between the presence of fQRS and all-cause or arrhythmic mortality. Also the rate of appropriate ICD shocks were similar among non-fQRS and fQRS groups. Additionally, in a retrospective study, Forleo et al. ${ }^{10}$ found that fQRS did not predict the arrhythmic events and mortality. The discussed study findings may be negatively resulted for fQRS due to heterogenous study populations in which ICD recipients consisted of primary and secondary prophylaxis groups. However, contrary to previous study results, Micheal et al. ${ }^{11}$ and Sha et al. ${ }^{12}$ have showed that there were significant association of fQRS with ventricular arrhythmias and mortality in patients with nonischemic cardiomyopathy. Also, Maskoun et al. ${ }^{13}$ reported that fQRS as a predictor of arrhythmic events in patients with ischemic cardiomyopathy. Similarly, in a recently published retrospective study by Brenyo et al. ${ }^{26}$ they confirmed the relationship of fQRS with appropriate ICD shocks and sudden cardiac death in patients with ischemic cardiomyopathy. So, there were conflicting data regarding the prognostic role of fQRS in patients with systolic heart failure. Our study results were consistent with the significant prognostic role of fQRS in either ischemic or nonischemic cardiomyopathy patients who underwent ICD implantation for primary prophylaxis. We have showed that fQRS was associated with appropriate ICD shocks for malignant ventricular arrhythmias, but was not associated with all-cause mortality. Additionally, the number of leads with fQRS found as significant predictor for appropriate ICD shocks. Because of well documented negative correlation of LVEF with the number of leads with fQRS, we proposed that those patients with higher number of leads with fQRS had greater myocardial scar tissue in our study. Also, we assumed that more leads with fQRS reflecting increased myocardial scar tissue was well correlated with appropriate ICD shocks.

Our study should be evaluated by some limitations. First, it is a cross-sectional study with small sample size, so prospectively designed large scale studies may precisely highlight the association of fQRS with arrhythmic events. Second, quantification of the myocardial scar tissue by SPECT or cardiac MRI may well define the association of fQRS with arrhythmic events.

In conclusion, fQRS which is a simple, inexpensive and readily available standard 12-lead ECG marker, predicts arrhythmic events and appropriate ICD shocks, but not all-cause mortality in patients with systolic heart failure undergoing ICD implantation for primary prophylaxis. So, fQRS may be used as an additional noninvasive marker for risk stratification and patient selection before ICD implantation for primary prophylaxis.

\section{REFERENCES}

1. Yancy CW, Jessup M, Bozkurt B, et al. 2013 ACCF/AHA Guideline for the management of heart failure: a report of the American College of Cardiology Foundation/American Heart Association Task Force on Practice Guidelines. J Am Coll Cardiol 2013;62(16):e147-e239.

2. Chow T, Kereiakes DJ, Bartone C, et al. Microvolt T-wave alternans identifies patients with ischemic cardiomyopathy who benefit from implantable cardioverter-defibrillator therapy. J Am Coll Cardiol 2007;49:50-58.

3. Zipes DP, Camm AJ, Borggrefe M, et al.; American College of Cardiology/American Heart Association Task Force, European Society of Cardiology Committee for Practice 
Guidelines, European Heart Rhythm Association, Heart Rhythm Society. ACC/AHA/ESC 2006 Guidelines for Management of Patients With Ventricular Arrhythmias and the Prevention of Sudden Cardiac Death: a report of the American College of Cardiology/American Heart Association Task Force and the European Society of Cardiology Committee for Practice Guidelines (writing committee to develop Guidelines for Management of Patients With Ventricular Arrhythmias and the Prevention of Sudden Cardiac Death): developed in collaboration with the European Heart Rhythm Association and the Heart Rhythm Society. Circulation 2006;114:e385-484.

4. Das MK, Suradi H, Maskoun W, et al. Fragmented wide QRS on a 12-lead ECG: a sign of myocardial scar and poor prognosis. Circ Arrhythm Electrophysiol 2008;1:258268.

5. Das MK, Saha C, El Masry H, et al. Fragmented QRS on a 12-lead ECG: a predictor of mortality and cardiac events in patients with coronary artery disease. Heart Rhythm 2007;4:1385-1392.

6. Pietrasik G, Goldenberg I, Zdzienicka J, et al. Prognostic significance of fragmented QRS complex for predicting the risk of recurrent cardiac events in patients with Qwave myocardial infarction. Am J Cardiol 2007;100:583586.

7. Canpolat U, Kabakçi G, Aytemir K, et al. Fragmented QRS complex predicts the arrhythmic events in patients with arrhythmogenic right ventricular cardiomyopathy/dysplasia. J Cardiovasc Electrophysiol 2013;24:12601266.

8. Das MK, Maskoun W, Shen C, et al. Fragmented QRS on twelve-lead electrocardiogram predicts arrhythmic events in patients with ischemic and nonischemic cardiomyopathy. Heart Rhythm 2010;7:74-80.

9. Cheema A, Khalid A, Wimmer A, et al. Fragmented QRS and mortality risk in patients with left ventricular dysfunction. Circ Arrhythm Electrophysiol 2010;3:339344.

10. Forleo GB, Della Rocca DG, Papavasileiou LP, et al. Predictive value of fragmented QRS in primary prevention implantable cardioverter defibrillator recipients with left ventricular dysfunction. J Cardiovasc Med 2011;12:779784.

11. Michael M, Das MK. Fragmented QRS (fQRS) on 12-lead EKG is a predictor of arrhythmic events and mortality in patients with dilated cardiomyopathy. Heart Rhythm 2006;(3 Suppl):S103.

12. Sha J, Zhang S, Tang M, et al. Fragmented QRS is associated with all-cause mortality and ventricular arrhythmias in patient with idiopathic dilated cardiomyopathy. Ann Noninvasive Electrocardiol 2011;16:270-275.

13. Maskoun W, Suradi H, Mahenthiran J, et al. Fragmented QRS complexes on a 12- lead ECG predict arrhythmic events in patients with ischemic cardiomyopathy who receive an ICD for primary prophylaxis. Heart Rhythm 2007;4:S211S212.
14. Das MK, Khan B, Jacob S, et al. Significance of a fragmented QRS complex versus a $\underline{Q}$ wave in patients with coronary artery disease. Circulation 2006;113:2495-2501.

15. Lang RM, Bierig M, Devereux RB, et al.; Chamber Quantification Writing Group, American Society of Echocardiography's Guidelines, Standards Committee, European Association of Echocardiography. Recommendations for chamber quantification: a report from the American Society of Echocardiography's Guidelines and Standards Committee and the Chamber Quantification Writing Group, developed in conjunction with the European Association of Echocardiography, a branch of the European Society of Cardiology. J Am Soc Echocardiogr 2005;18:1440-1463.

16. Mahenthiran J, Khan BR, Sawada SG, et al. Fragmented QRS complexes not typical of a bundle branch block: a marker of greater myocardial perfusion tomography abnormalities in coronary artery disease. J Nucl Cardiol 2007;14(3):347-353.

17. Varriale $\mathrm{P}, \mathrm{Chryssos} \mathrm{BE}$. The RSR' complex not related to right bundle branch block: diagnostic value as a sign of myocardial infarction scar. Am Heart J 1992;123:369-376.

18. Flowers NC, Horan LG, Thomas JR, et al. The anatomic basis for highfrequency components in the electrocardiogram. Circulation 1969;39:531-539.

19. Homsi M, Alsayed L, Das MK, et al. Fragmented QRS complexes on a 12-lead ECG is a marker of greater myocardial scarring related to coronary artery disease by magnetic resonance imaging. J Am Coll Cardiol 2008;51:A31.

20. Homsi M, Alsayed L, Das MK, et al. Fragmented QRS complexes on a 12- lead ECG is a marker of greater myocardial scarring related to non-coronary artery diseases by magnetic resonance imaging. J Am Coll Cardiol 2009;53:A140.

21. Basaran $Y$, Tigen $K$, Karaahmet $T$, et al. Fragmented QRS complexes are associated with cardiac fibrosis and significant intraventricular systolic dyssynchrony in nonischemic dilated cardiomyopathy patients with a narrow QRS interval. Echocardiography 2011;28:62-68.

22. Park SJ, On YK, Kim JS, et al. Relation of fragmented QRS complex to right ventricular fibrosis detected by late gadolinium enhancement cardiac magnetic resonance in adults with repaired tetralogy of fallot. Am J Cardiol 2012;109:110-115.

23. Homsi M, Alsayed L, Safadi B, et al. Fragmented QRS complexes on 12-lead ECG: a marker of cardiac sarcoidosis as detected by gadolinium cardiac magnetic resonance imaging. Ann Noninvasive Electrocardiol 2009;14:319-326.

24. Stevenson WG, Soejima K. Catheter ablation for ventricular tachycardia. Circulation 2007;115:2750-2760.

25. Wiener I, Mindich B, Pitchon R. Fragmented endocardial electrical activity in patients with ventricular tachycardia: a new guide to surgical therapy. Am Heart J 1984;107:86-90.

26. Brenyo A, Pietrasik G, Barsheshet A, et al. QRS fragmentation and the risk of sudden cardiac death in MADIT II. J Cardiovasc Electrophysiol 2012;23:1343-1348. 